Filomat 28:7 (2014), 1315-1322

DOI 10.2298/FIL1407315A

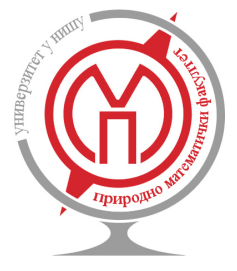

Published by Faculty of Sciences and Mathematics, University of Niš, Serbia

Available at: http://www.pmf.ni.ac.rs/filomat

\title{
Graphs with Maximal Irregularity
}

\author{
Hosam Abdo ${ }^{\mathrm{a}}$, Nathann Cohen ${ }^{\mathrm{b}}$, Darko Dimitrov ${ }^{\mathrm{a}}$ \\ ${ }^{a}$ Institut für Informatik, Freie Universität Berlin, Takustraße 9, D-14195 Berlin, Germany \\ ${ }^{b}$ Algorithms Research Group, Computer Science Dept., Université Libre de Bruxelles, 1050 Brussels, Belgium
}

\begin{abstract}
Albertson [3] has defined the irregularity of a simple undirected graph $G=(V, E)$ as $\operatorname{irr}(G)=$ $\sum_{u v \in E}\left|d_{G}(u)-d_{G}(v)\right|$, where $d_{G}(u)$ denotes the degree of a vertex $u \in V$. Recently, this graph invariant gained interest in the chemical graph theory, where it occured in some bounds on the first and the second Zagreb index, and was named the third Zagreb index [12]. For general graphs with $n$ vertices, Albertson has obtained an asymptotically tight upper bound on the irregularity of $4 n^{3} / 27$. Here, by exploiting a different approach than in [3], we show that for general graphs with $n$ vertices the upper bound $\left\lfloor\frac{n}{3}\right\rfloor\left\lceil\frac{2 n}{3}\right\rceil\left(\left\lceil\frac{2 n}{3}\right\rceil-1\right)$ is sharp. We also present lower bounds on the maximal irregularity of graphs with fixed minimal and/or maximal vertex degrees, and consider an approximate computation of the irregularity of a graph.
\end{abstract}

\section{Introduction}

Let $G=(V, E)$ be a simple undirected graph of order $n=|V|$ and size $m=|E|$. For $v \in V(G)$, the degree of $v$, denoted by $d_{G}(v)$, is the number of edges incident to $v$. Albertson [3] defines the imbalance of an edge $e=u v \in E$ as $\left|d_{G}(u)-d_{G}(v)\right|$ and the irregularity of $G$ as

$$
\operatorname{irr}(G)=\sum_{u v \in E}\left|d_{G}(u)-d_{G}(v)\right|
$$

The first Zagreb index $M_{1}(G)$ and the second Zagreb index $M_{2}(G)$, are one of the oldest and most investigated topological graph indices, and are defined as follows:

$$
\begin{aligned}
& M_{1}(G)=\sum_{v \in V} d_{G}(v)^{2}, \\
& M_{2}(G)=\sum_{u v \in E} d_{G}(u) d_{G}(v) .
\end{aligned}
$$

For details of the mathematical theory and chemical applications of the Zagreb indices see surveys [10, 13, $18,23]$ and papers $[11,12,24-26]$.

2010 Mathematics Subject Classification. 05C07; 90C35, 05C90

Keywords. irregularity of a graph, Zagreb indices, third Zagreb index

Received: 01 September 2012 ; Accepted: 13 September 2013

Communicated by Dragan Stevanović

Email addresses: abdo@inf. fu-berlin.de (Hosam Abdo), nathann. cohen@gmail .com (Nathann Cohen), darko@inf.fu-berlin.de (Darko Dimitrov) 
Recently in [12], Fath-Tabar established new bounds on the first and the second Zagreb indices which depend on the sum in (1). In line with the standard terminology of chemical graph theory, and the obvious connection with the first and the second Zagreb indices, Fath-Tabar named the sum in (1) the third Zagreb index and denoted it by $M_{3}(G)$. However, in the rest of the paper, we will use its older name and call it the irregularity of a graph.

Obviously, a connected graph $G$ has irregularity zero if and only if $G$ is regular. Other approaches, that characterize how irregular a graph is, have been proposed $[1,2,6-9,14]$. In this paper, we focus on graphs with maximal irregularity as defined in (1).

In [3] Albertson presented upper bounds on irregularity for bipartite graphs, triangle-free graphs and arbitrary graphs, as well as a sharp upper bound for trees. Some claims about bipartite graphs given in [3] have been formally proved in [16]. Related to Albertson [3] is the work of Hansen and Mélot [15], who characterized the graphs with $n$ vertices and $m$ edges with maximal irregularity. For more results on irregularity, imbalance, and related measures, we redirect the reader to [2, 4, 5, 19-21].

In the sequel we introduce the notation used in the rest of the paper.

A regular graph is a graph where all the vertices have the same degree. A pendant vertex is a vertex of degree one. A universal vertex is the vertex adjacent to all other vertices. The diameter of a graph $G$ is the maximal distance between any two vertices of $G$. A set of vertices is said to be independent when the vertices are pairwise non-adjacent. The vertices from an independent set are independent vertices. By $N_{G}(u)$, we denote the set of vertices that are adjacent to a vertex $u$.

A clique of a graph $G$ is a complete subgraph of $G$. The union $G=G_{1} \cup G_{2}$ of graphs $G_{1}$ and $G_{2}$ with disjoint vertex sets $V_{1}$ and $V_{2}$ and edge sets $E_{1}$ and $E_{2}$ is the graph with the vertex set $V=V_{1} \cup V_{2}$ and the edge set $E=E_{1} \cup E_{2}$. The join $G=G_{1}+G_{2}$ of the graphs $G_{1}$ and $G_{2}$ is the graph union $G=G_{1} \cup G_{2}$ together with all the edges joining $V_{1}$ and $V_{2}$.

The clique-star graph $K S_{p, q}$ is the join graph of a clique of size $p$ and an independent set of size $q$ (see Fig.1).

\section{General Graphs with Maximal Irregularity}

In order to characterize graphs with maximal irregularity, we first determine the minimum number of universal vertices that such graphs must have.

Lemma 2.1. Let $G$ be a graph with maximal irregularity among all graphs of order $n$. Then, $G$ has at least $\left\lfloor\frac{n}{3}\right\rfloor$ universal vertices.

Proof. Assume that $G$ is a graph with maximal irregularity whose set $U$ of universal vertices has cardinality $q<\left\lfloor\frac{n}{3}\right\rfloor$. Let $\bar{U}=\left\{\bar{u}_{1}, \ldots, \bar{u}_{n-q}\right\}$ be the set of non-universal vertices, where $d\left(\bar{u}_{1}\right) \geq d\left(\bar{u}_{2}\right) \geq \cdots \geq d\left(\bar{u}_{n-q-1}\right) \geq$ $d\left(\bar{u}_{n-q}\right)$.

If a non-neighbor $x$ of $\bar{u}_{1}$ is adjacent to a vertex $y \in \bar{U} \cap N_{G}\left(\bar{u}_{1}\right)$, then replace the edge $x y$ with the edge $\bar{u}_{1} x$, obtaining a graph $G^{\prime}$. By this replacement, the number of edges remains the same, as well as the degree of $x$. Also, the contribution of the edges between the $q$ universal vertices and the vertices of $\bar{U}$ to $\operatorname{irr}\left(G^{\prime}\right)$ remains unchanged.

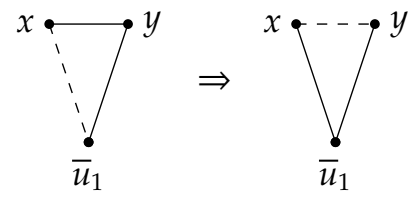

The contribution of the edge $x y$ to $\operatorname{irr}(G)$ is $\left|d_{G}(y)-d_{G}(x)\right|$, and the contribution of the edge $\bar{u}_{1} x$ to $\operatorname{irr}\left(G^{\prime}\right)$ is $d_{G}\left(\bar{u}_{1}\right)+1-d_{G}(x)$. After the above edge replacement, edges between $\bar{u}_{1}$ and $\bar{U} \cap N_{G}\left(\bar{u}_{1}\right)$ increase 
the irregularity by $d_{G}\left(\bar{u}_{1}\right)-q$, and edges between $y$ and $\bar{U} \backslash\left\{\bar{u}_{1}, x\right\}$ decrease the irregularity by at most $d_{G}(y)-q-2$. Thus,

$$
\begin{aligned}
\operatorname{irr}\left(G^{\prime}\right)= & \operatorname{irr}(G)-\overbrace{\left|d_{G}(y)-d_{G}(x)\right|}^{x y}+\overbrace{d_{G}\left(\bar{u}_{1}\right)+1-d_{G}(x)}^{\bar{u}_{1} x} \\
& +\underbrace{d_{G}\left(\bar{u}_{1}\right)-q}_{\text {edges from } \bar{u}_{1} \text { to } \bar{u} \cap N_{G}\left(\bar{u}_{1}\right)} \underbrace{-d_{G}(y)+q+2}_{\text {edges from } y \text { to } \bar{u} \backslash\left\langle\bar{u}_{1}, x\right\}} \\
= & \operatorname{irr}(G) \underbrace{-\left|d_{G}(y)-d_{G}(x)\right|-d_{G}(x)-d_{G}(y)}_{-2 \max \left(d_{G}(x), d_{G}(y)\right)}+2 d_{G}\left(\bar{u}_{1}\right)+3 \\
= & \operatorname{irr}(G)+2\left(d_{G}\left(\bar{u}_{1}\right)-\max \left(d_{G}(x), d_{G}(y)\right)\right)+3 .
\end{aligned}
$$

Since $d_{G}\left(\bar{u}_{1}\right) \geq d_{G}(x), d_{G}(y)$, it follows that $\operatorname{irr}\left(G^{\prime}\right)>\operatorname{irr}(G)$. We apply the above kind of replacement for all edges between $\bar{U} \cap N_{G}\left(\bar{u}_{1}\right)$ and $\bar{U} \backslash N_{G}\left(\bar{u}_{1}\right)$ - which only increases the irregularity of $G_{1}$ - so that we can now assume that there are none. Note also that $\bar{u}_{1}$ cannot have become an universal vertex, as it would contradict the assumption that a graph with maximal irregularity has at most $q$ universal vertices. Therefore, $\bar{u}_{1}$ is still the vertex of $\bar{U}$ of maximal degree. We denote by $G_{1}$ the newly obtained graph.

Next, we replace any edge $x y$ between two vertices $x, y \in \bar{U} \backslash N_{G_{1}}\left(\bar{u}_{1}\right)$ by $\bar{u}_{1} x$ - this replacement preserves the number of edges as well the degree of $x$. The newly obtained graph we denote by $G^{\prime \prime}$. The contribution of the edges between the $q$ universal vertices and the vertices of $\bar{U}$ to $\operatorname{irr}\left(G_{1}\right)$ are unchanged. The contribution

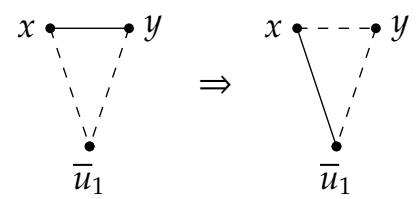

of edge $x y$ to $\operatorname{irr}\left(G_{1}\right)$ is $\left|d_{G_{1}}(y)-d_{G_{1}}(x)\right|$, and the contribution of the edge $\bar{u}_{1} x$ to $\operatorname{irr}\left(G^{\prime \prime}\right)$ is $d_{G_{1}}\left(\bar{u}_{1}\right)+1-d_{G_{1}}(x)$. Edges between $\bar{u}_{1}$ and $\bar{U} \cap N_{G}\left(\bar{u}_{1}\right)$ increase the irregularity by $d_{G_{1}}\left(\bar{u}_{1}\right)-q$, and edges between $y$ and $\bar{u} \backslash N_{G}\left(\bar{u}_{1}\right) \backslash\{u\}$ decrease the irregularity by at most $d_{G_{1}}(y)-q-1$. Therefore,

$$
\begin{aligned}
\operatorname{irr}\left(G^{\prime \prime}\right) \geq & \operatorname{irr}\left(G_{1}\right)-\overbrace{\left|d_{G_{1}}(y)-d_{G_{1}}(x)\right|}^{x y}+\overbrace{d_{G_{1}}\left(\bar{u}_{1}\right)+1-d_{G_{1}}(x)}^{\bar{u}_{1} x} \\
& +\underbrace{d_{G_{1}}\left(\bar{u}_{1}\right)-q}_{\text {edges from } \bar{u}_{1} \text { to } \bar{u} \cap N_{G_{1}}\left(\bar{u}_{1}\right)} \quad \underbrace{-d_{G_{1}}(y)+q+1}_{\text {edges from } y \text { to } \bar{u} \backslash N_{G_{1}}\left(\bar{u}_{1}\right) \backslash\{x\}} \\
= & \operatorname{irr}\left(G_{1}\right) \underbrace{-\left|d_{G_{1}}(y)-d_{G_{1}}(x)\right|-d_{G_{1}}(y)-d_{G_{1}}(x)}_{-2 \max \left(d_{G_{1}}(y), d_{G_{1}}(x)\right)}+2 d_{G_{1}}\left(\bar{u}_{1}\right)+2 \\
= & \operatorname{irr}\left(G_{1}\right)+2\left(d_{G_{1}}\left(\bar{u}_{1}\right)-\max \left(d_{G_{1}}(y), d_{G_{1}}(x)\right)+2 .\right.
\end{aligned}
$$

As $d_{G_{1}}\left(\bar{u}_{1}\right) \geq d_{G_{1}}(y), d_{G_{1}}(x)$, we have that $\operatorname{irr}\left(G^{\prime \prime}\right)>\operatorname{irr}\left(G_{1}\right)$. Hence, we can apply this second replacement to all edges between vertices of $\bar{U} \backslash N_{G_{1}}\left(\bar{u}_{1}\right)$ - which only increases $\operatorname{irr}(G)$ - and so assume that there are none. We denote by $G_{2}$ the newly obtained graph. As previously, $\bar{u}_{1}$ cannot become universal because of this 
procedure - that would contradict our assumptions on $G$. Thus, $\bar{U} \backslash N_{G}\left(\bar{u}_{1}\right)$ is a nonempty independent set whose cardinality we note $z>0$.

We can build a new graph $G^{\star}$ with $q+1$ universal vertices from $G_{2}$, by linking $\bar{u}_{1}$ to its $z$ non-neighbor. As this operation changes the degree of $z+1$ vertices, the contribution of the edges between the $q$ universal vertices and the rest of the vertices to $\operatorname{irr}\left(G^{\star}\right)$ is by $2 z q$ smaller than their contribution to $\operatorname{irr}\left(G_{2}\right)$. However, The $z$ new edges between $\bar{u}_{1}$ and $\bar{U} \cap N_{G_{2}}\left(\bar{u}_{1}\right)$ contributes $z(n-1-q-1)$ to $\operatorname{irr}\left(G^{\star}\right)$, and the contribution between $\bar{u}_{1}$ and vertices of $\bar{U} \cap N_{G_{2}}\left(\bar{u}_{1}\right)$ increases in $\operatorname{irr}\left(G^{\star}\right)$ by $z(n-q-z-1)$. Therefore,

$$
\begin{aligned}
\operatorname{irr}\left(G^{\star}\right) & =\operatorname{irr}\left(G_{2}\right)-2 z q+z(n-1-q-1)+z(n-q-z-1) \\
& =\operatorname{irr}\left(G_{2}\right)+z(2 n-4 q-z-3) .
\end{aligned}
$$

As $z \leq n-q-1$, further we have

$$
\operatorname{irr}\left(G^{\star}\right) \geq \operatorname{irr}\left(G_{2}\right)+z(n-3 q-2) .
$$

Since we have assumed $q<\left\lfloor\frac{n}{3}\right\rfloor$, it follows that $\operatorname{irr}\left(G^{\star}\right)>\operatorname{irr}\left(G_{2}\right)$. Thus, we have shown that we can obtain a graph $G^{\star}$ with $q+1$ universal vertices with irregularity greater than any graph $G$ with maximal irregularity, which is a contradiction to the assumption that any graph $G$ with maximal irregularity has at most $q$ universal vertices.

We will now determine the graphs whose irregularity is maximum.

Theorem 2.2. If a graph $G$ has maximal irregularity among all graphs of order $n$, then $G$ is either the clique-star graph $K S_{\left.\left\lfloor\frac{n}{3}\right\rfloor, \frac{2 n}{3}\right]}$, or, if $n \equiv 2(\bmod 3)$, the clique-star graph $K S_{\left\lceil\frac{n}{3}\right\rceil,\left\lfloor\frac{2 n}{3}\right\rfloor}$.

Proof. Let $G$ be a graph of maximum irregularity, and let $U=\left\{v_{n-q+1}, v_{n-q+2}, \ldots, v_{n}\right\}$ be the set of universal vertices, where $q \geq\left\lfloor\frac{n}{3}\right\rfloor$ (cf. Lemma 2.1). Let $\bar{U}$ be the set of non-universal vertices, let $G[\bar{U}]$ be the graph induced by all non-universal vertices, and let $G^{\prime}=G-G[\bar{U}]$ be the complement of $G[\bar{U}]$ in $G$.

As $d_{G^{\prime}}(v)=d_{G}(v)-d_{\bar{U}}(v)=q$, the edges between $U$ and $\bar{U}$ contribute more to $\operatorname{irr}\left(G^{\prime}\right)$ than they do to $\operatorname{irr}(G)$, by a difference of

$$
\sum_{v \in \bar{U}} d_{\bar{U}}(v) q
$$

On the other hand, the contribution of edges from $G[\bar{U}]$ does not appear in the computation of $\operatorname{irr}\left(G^{\prime}\right)$. The difference of the degrees between the endvertices of an edge of $G[\bar{U}]$ is at most $n-q-3$. Therefore, the edges from $G[\bar{U}]$ contribute to $\operatorname{irr}(G)$ by most

$$
\frac{1}{2} \sum_{v \in \bar{U}} d_{\bar{U}}(v)(n-q-3)
$$

From (4) and (5), we have

$$
\begin{aligned}
\operatorname{irr}\left(G^{\prime}\right) & \geq \operatorname{irr}(G)+\sum_{v \in \bar{U}} d_{\bar{U}}(v) q-\frac{1}{2} \sum_{v \in \bar{U}} d_{\bar{U}}(v)(n-q-3) \\
& =\operatorname{irr}(G)+\left(\frac{1}{2}(3 q-n)+\frac{3}{2}\right) \sum_{v \in \bar{U}} d_{\bar{U}}(v) .
\end{aligned}
$$

The expression $\frac{1}{2}(3 q-n)+\frac{3}{2}$ is positive for $q \geq\left\lfloor\frac{n}{3}\right\rfloor$. Since, $G$ is a graph with maximal irregularity, it follows that $\sum_{v \in \bar{U}} d_{\bar{U}}(v)=0$, i.e., the vertices of $\bar{U}$ form an independent set. Therefore, $G$ is a clique-star graph $K S_{q, n-q}$, with $q \geq\left\lfloor\frac{n}{3}\right\rfloor$. The irregularity of $K S_{q, n-q}$ is $q(n-q)(n-1-q)$, and it is maximized for $q=\left\lfloor\frac{n}{3}\right\rfloor$, and for $q=\left\lceil\frac{n}{3}\right\rceil$, if $n \equiv 2(\bmod 3)$.

The graphs with maximal irregularity with 6, 7 and 8 vertices are depicted in Figure 1. 


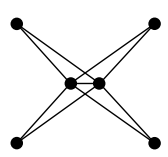

(a)

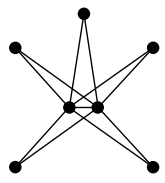

(b)

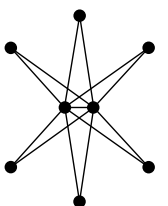

(c)

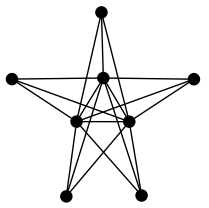

$(d)$

Figure 1: (a) The graph with 6 vertices with maximal irr. (b)The graph with 7 vertices with maximal irr. (c) and (d) Graphs with 8 vertices with maximal irr.

Corollary 2.3. For any $G, \operatorname{irr}(G) \leq\left\lfloor\frac{n}{3}\right\rfloor\left\lceil\frac{2 n}{3}\right\rceil\left(\left\lceil\frac{2 n}{3}\right\rceil-1\right)=\operatorname{irr}\left(K S_{\left\lfloor\frac{n}{3}\right\rfloor,\left\lceil\frac{2 n}{3}\right\rceil}\right)$.

\section{Lower Bounds on Graphs with Maximal Irregularity}

In this section, we consider graphs with maximal irregularity and prescribed minimal or/and maximal degrees. First, we show a lower bound for graphs with fixed maximal degree $\Delta$.

Proposition 3.1. Let $G$ be a connected graph with $n$ vertices with maximum degree $\Delta(G)=\Delta$, and maximal irregularity. Then, it holds that

$$
\operatorname{irr}(G) \geq \frac{4 \Delta^{2} n}{27}+n O(\Delta)
$$

Proof. To obtain the bound we consider the graph $Q$ which is illustrated in Figure 2.
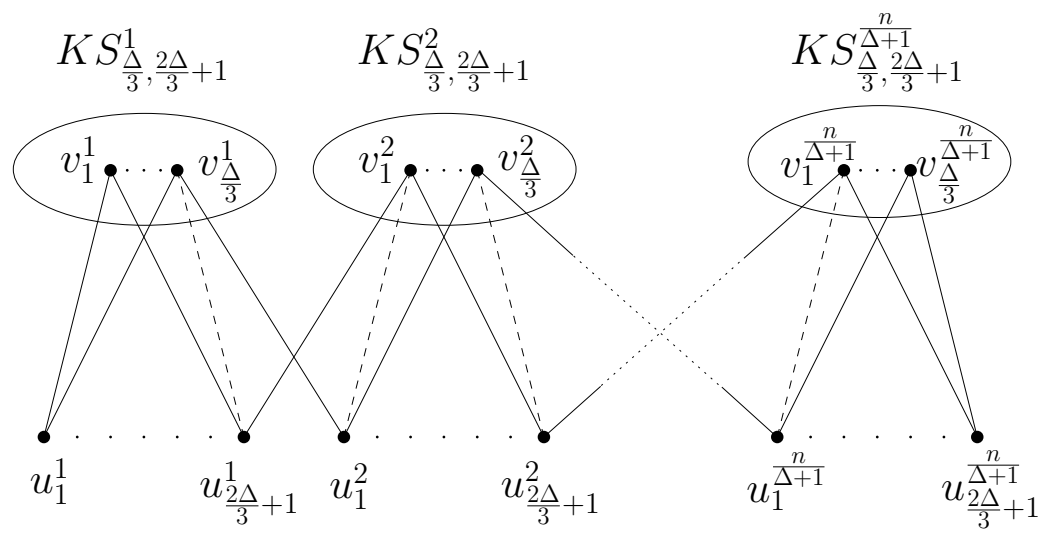

Figure 2: A connected graph $Q$ constructed from $n /(\Delta+1)$ copies of $K S_{\frac{\Delta}{3}, \frac{2 \Delta}{3}+1}$. The dashed edges are those that are removed from the corresponding clique-star graphs.

To simplify the calculation, we assume that $\Delta / 3$ and $n /(\Delta+1)$ are integers. The construction of $Q$ is as follows:

- Make a sequence of $n /(\Delta+1)$ copies of $K S_{\frac{\Delta}{3}, \frac{2 \pi}{3}+1}$.

- Choose an edge from the first $K S_{\frac{\Delta}{3}, \frac{2 \Delta}{3}+1}$ graph, with an independent vertex as one endvertex, and an edge from the second $K S_{\frac{\Delta}{3}, \frac{2 \Delta}{3}+1}$ graph, also with an independent vertex as one endvertex. Let 
denote these edges by $v_{\frac{\Delta}{3}}^{1} u_{\frac{2 \Delta}{3}+1}^{1}$ and $v_{1}^{2} u_{1}^{2}$, respectively. Replace $v_{\frac{\Delta}{3}}^{1} u_{\frac{2 \Delta}{3}+1}^{1}$ and $v_{1}^{2} u_{1}^{2}$ by edges $v_{\frac{\Delta}{3}}^{1} u_{1}^{2}$ and $v_{1}^{2} u_{\frac{2 \Delta}{3}+1}^{1}$. Continue this kind of replacement between all consecutive copies of $K S_{\frac{1}{3}, \frac{2 \Delta}{3}+1}$. Notice that these replacements do not change the degrees of the vertices.

We have

$$
\operatorname{irr}(Q)=\frac{n}{\Delta+1} \frac{\Delta}{3}\left(\frac{2 \Delta}{3}+1\right) \frac{2 \Delta}{3}=\frac{4 \Delta^{2} n}{27}+n O(\Delta)
$$

Next, we show a lower bound for graphs with maximal irregularity and fixed minimal degree $\delta$.

Proposition 3.2. Let $G$ be a connected graph with $n$ vertices with minimal degree $\delta(G)=\delta$, and maximal irregularity. Then

$$
\operatorname{irr}(G) \geq \delta(n-\delta)(n-1-\delta)=\Omega\left(\delta n^{2}\right) .
$$

Proof. The lower bound is obtained by $K S_{\delta, n-\delta}$ whose irregularity is $\delta(n-\delta)(n-1-\delta)$.

Finally, we show a lower bound for graphs with maximal irregularity and fixed maximal and minimal degrees.

Proposition 3.3. Let $G$ be a connected graph with $n$ vertices with minimal degree $\delta(G)=\delta$, maximal degree $\Delta(G)=\Delta$, and maximal irregularity. Then, it holds

$$
\operatorname{irr}(G)>\frac{\delta}{\Delta+1}(\Delta-\delta)^{2} n .
$$

Proof. To obtain the bound we consider the graph $R$, which is constructed in the same way as the graph $Q$ in Figure 2, with only difference that $R$ is built of $n /(\Delta+1)$ copies of $K S_{\delta, \Delta-\delta+1}$. To simplify the calculation, we assume that $n /(\Delta+1)$ is integer. We have

$$
\operatorname{irr}(R)=\frac{n}{\Delta+1} \delta(\Delta-\delta+1)(\Delta-\delta) .
$$

\section{Computation of the Irregularity of a Graph}

In order to better understand the properties of graphs with large irregularity, we thought sensible to approach the problem by enumerating the graphs with a fixed number $n$ of vertices in order to compute the irregularity of each of them. While such a procedure is made easy by the software Sage [22], or Brendan McKay's Nauty [17], the exhaustive enumeration of graphs quickly becomes impractical due to the sheer number of such graphs (which happens in practice as soon as $n \approx 11$ ). Therefore, we attempted to relax the computational problem by enumerating the possible degree sequences of graphs with a fixed number of vertices instead of the graphs themselves. Indeed, the number of different degree sequences of graphs with $n$ vertices is fairly small compared to the number of non-isomorphic graphs, and the code necessary to enumerate them much simpler ${ }^{1}$.

One can not hope, however, to compute the value of irr with only a degree sequence, though it is possible to upper-bound the irregularity of a graph $G$ with this information. The following lines describe a bound on the irregularity of a graph depending only on its degree sequence.

$$
\operatorname{irr}(G)=\sum_{u v \in E(G)}|d(u)-d(v)|
$$

\footnotetext{
${ }^{1)}$ Our implementation is included in the software package Sage [22].
} 


$$
=\sum_{0 \leq i<n} \mid\{u v \in E(G): d(u) \leq i \text { and } d(v)>i\} \mid .
$$

Let us now write $d_{\leq i}$ (resp. $d_{>i}$ ) the number of vertices of $G$ whose degree is smaller (resp. strictly larger) than $i$. Given a vertex $v$ of degree $\leq i$, the number of neighbors of degree $>i$ it can have is necessarily smaller than both $d_{>i}$ and $d(v)$. Following the same steps for vertices $v$ of degree $>i$, we obtain

$$
\operatorname{irr}(G) \leq \sum_{0 \leq i<n} \min \left[\sum_{\substack{v \in V(G) \\ d(v) \leq i}} \min \left(d(v), d_{>i}\right), \sum_{\substack{v \in V(G) \\ d(v)>i}} \min \left(d(v), d_{\leq i}\right)\right] .
$$

By computing this bound on all the degree sequences of graphs with $n$ vertices, we obtained the list of the

\begin{tabular}{||l|l|l|c||}
\hline \hline$n$ & $K S_{n}^{\max }$ & graphic sequences of $K S_{n}^{\max }$ & $\operatorname{irr}\left(K S_{n}^{\max }\right)$ \\
\hline \hline 3 & $K S_{1,2}$ & {$[2,1,1]$} & 2 \\
4 & $K S_{1,3}$ & {$[3,1,1,1]$} & 6 \\
5 & $K S_{1,4}$ & {$[4,1,1,1,1]$} & 12 \\
& $K S_{2,3}$ & {$[4,4,2,2,2]$} & 12 \\
6 & $K S_{2,4}$ & {$[5,5,2,2,2,2]$} & 24 \\
7 & $K S_{2,5}$ & {$[6,6,2,2,2,2,2]$} & 40 \\
8 & $K S_{2,6}$ & {$[7,7,2,2,2,2,2,2]$} & 60 \\
& $K S_{3,5}$ & {$[7,7,7,3,3,3,3,3]$} & 60 \\
9 & $K S_{3,6}$ & {$[8,8,8,3,3,3,3,3,3]$} & 90 \\
10 & $K S_{3,7}$ & {$[9,9,9,3,3,3,3,3,3,3]$} & 126 \\
11 & $K S_{3,8}$ & {$[10,10,10,3,3,3,3,3,3,3,3]$} & 168 \\
& $K S_{4,7}$ & {$[10,10,10,10,4,4,4,4,4,4,4]$} & 168 \\
12 & $K S_{4,8}$ & {$[11,11,11,11,4,4,4,4,4,4,4,4]$} & 224 \\
13 & $K S_{4,9}$ & {$[12,12,12,12,4,4,4,4,4,4,4,4,4]$} & 288 \\
14 & $K S_{4,10}$ & {$[13,13,13,13,4,4,4,4,4,4,4,4,4,4]$} & 360 \\
& $K S_{5,9}$ & {$[13,13,13,13,13,5,5,5,5,5,5,5,5,5]$} & 360 \\
15 & $K S_{5,10}$ & {$[14,14,14,14,14,5,5,5,5,5,5,5,5,5,5]$} & 450 \\
16 & $K S_{5,11}$ & {$[15,15,15,15,15,5,5,5,5,5,5,5,5,5,5,5]$} & 550 \\
17 & $K S_{5,12}$ & {$[16,16,16,16,16,5,5,5,5,5,5,5,5,5,5,5,5]$} & 660 \\
& $K S_{6,11}$ & {$[16,16,16,16,16,16,6,6,6,6,6,6,6,6,6,6,6]$} & 660 \\
18 & $K S_{6,12}$ & {$[17,17,17,17,17,17,6,6,6,6,6,6,6,6,6,6,6,6]$} & 792 \\
19 & $K S_{6,13}$ & {$[18,18,18,18,18,18,6,6,6,6,6,6,6,6,6,6,6,6,6]$} & 936 \\
20 & $K S_{6,14}$ & {$[19,19,19,19,19,19,6,6,6,6,6,6,6,6,6,6,6,6,6,6]$} & 1092 \\
& $K S_{7,13}$ & {$[19,19,19,19,19,19,19,7,7,7,7,7,7,7,7,7,7,7,7,7]$} & 1092 \\
21 & $K S_{7,14}$ & {$[20,20,20,20,20,20,20,7,7,7,7,7,7,7,7,7,7,7,7,7,7]$} & 1274 \\
22 & $K S_{7,15}$ & {$[21,21,21,21,21,21,21,7,7,7,7,7,7,7,7,7,7,7,7,7,7,7]$} & 1470 \\
23 & $K S_{7,16}$ & {$[22,22,22,22,22,22,22,7,7,7,7,7,7,7,7,7,7,7,7,7,7,7,7]$} & 1680 \\
& $K S_{8,15}$ & {$[22,22,22,22,22,22,22,22,8,8,8,8,8,8,8,8,8,8,8,8,8,8,8]$} & 1680 \\
\hline \hline
\end{tabular}

Table 1: Graphs with the maximal irregularity, their corresponding graphic sequences, and the values of their irregularities.

degree sequences for which the value reached by this bound is maximal. This would not have necessarily meant that a graph having such degree sequence would have the largest irregularity among all graphs with $n$ vertices - for it is only an upper bound on the irregularity of such a graph - though we remarked in this situation that the degree sequences for which this bound was the largest corresponded to the graphs described by Theorem 2.2. In particular, as for these graphs the upper bound is equal to the irregularity, those graphs are indeed the (only) extremal ones.

In Table 1 are gathered the results of our experiments up to $n=23$, where the number of degree sequences, in turn, became too large to continue further. It contains for each $n$ the degree sequences 
maximizing the bound (6), along with a corresponding graph for which irr is equal to the upper bound. $K S_{n}^{\max }$ denotes a graph with the maximal irregularity among all clique-star graphs with $n$ vertices. By Theorem 2.2, $K S_{n}^{\max }$ has the maximal irregularity among all graphs with $n$ vertices, and is determined by

$$
K S_{n}^{\max }= \begin{cases}K S_{\left\lfloor\frac{n}{3}\right\rfloor,\left\lceil\frac{2 n}{7}\right\rceil} \text { and } K S_{\left\lceil\frac{n}{3}\right\rceil,\left\lfloor\frac{2 n}{3}\right\rfloor}, & \text { if } n \equiv 2(\bmod 3), \\ K S_{\left\lfloor\frac{n}{3}\right\rfloor,\left\lceil\frac{2 n}{3}\right\rceil}, & \text { otherwise. }\end{cases}
$$

\section{Acknowledgment}

We would like to thank Ivan Gutman for rising the question about graphs with maximal third Zagreb index and for providing us with useful references regarding the subject considered in this paper.

\section{References}

[1] Y. Alavi, A. Boals, G. Chartrand, P. Erdős, O. R. Oellermann, k-path Irregular Graphs , Congr. Numer. 65 (1988) 201-210.

[2] Y. Alavi, G. Chartrand, F. R. K. Chung, P. Erdős, R. L. Graham, O. R. Oellermann, Highly Irregular Graphs , J. Graph Theory 11 (1987) 235-249.

[3] M. O. Albertson, The Irregularity of a Graph , Ars Comb. 46 (1997) 219-225.

[4] F. K. Bell, A Note on the Irregularity of Graphs , Linear Algebra Appl. 161 (1992) 45-54.

[5] Y. Caro, R. Yuster, Graphs with Large Variance, Ars Comb. 57 (2000) 151-162.

[6] G. Chartrand, P. Erdős, O. R. Oellermann, How to Define an Irregular Graph, Coll. Math. J. 19 (1988) $36-42$.

[7] G. Chartrand, K. S. Holbert, O. R. Oellermann, H. C. Swart, F-Degrees in Graphs , Ars Comb. 24 (1987) 133-148.

[8] L. Collatz, U. Sinogowitz, Spektren endlicher Graphen, Abh. Math. Sem. Univ. Hamburg 21 (1957) 63-77.

[9] D. Cvetković, P. Rowlinson, On Connected Graphs with Maximal Index , Publications de l'Institut Mathematique (Beograd) 44 (1988) 29-34.

[10] K. C. Das, I. Gutman, Some Properties of the Second Zagreb Index , MATCH Commun. Math. Comput. Chem. 52 (2004) $103-112$.

[11] T. Došlić, B. Furtula, A. Graovac, I. Gutman, S. Moradi, Z. Yarahmadi, On Vertex Degree Based Molecular Structure Descriptors , MATCH Commun. Math. Comput. Chem. 66 (2011) 613-626.

[12] G. H. Fath-Tabar, Old and New Zagreb Indices of Graphs, MATCH Commun. Math. Comput. Chem. 65 (2011) 79-84.

[13] I. Gutman, K. C. Das, The First Zagreb Index 30 Years After , MATCH Commun. Math. Comput. Chem. 50 (2004) 83-92.

[14] D. E. Jackson, R. Entringer, Totally Segregated Graphs , Congress. Numer. 55 (1986) 159-165.

[15] P. Hansen, H. Mélot Variable Neighborhood Search for Extremal Graphs 9. Bounding the Irregularity of a Graph , DIMACS Ser. Discrete Math. Theoret. Comput. Sci. 69 (1962) $253-264$.

[16] M. A. Henning, D. Rautenbach, On the Irregularity of Bipartite Graphs , Discrete Math. 307 (2007) 1467-1472.

[17] B. D. McKay. Nauty (version 2.4), Computer Science Dept., Australian National University, 2007.

[18] S. Nikolić, G. Kovačević, A. Miličević, N. Trinajstić, The Zagreb Indices 30 Years After , Croat. Chem. Acta 76 (2003) 113-124.

[19] D. Rautenbach, Propagation of Mean Degrees, Electr. J. Comb. 11 (2004) N11.

[20] D. Rautenbach, L. Volkmann, How Local Irregularity Gets Global in a Graph , J. Graph Theory 41 (2002) 18-23.

[21] D. Rautenbach, I. Schiermeyer, Extremal Problems for Imbalanced Edges, Graphs Comb. 22 (2006) 103-111.

[22] W. Stein, G. Furnish, M. Hansen, R. Miller, Y. Qiang, Sage: Open Source Mathematical Software: Symbolic Computation, Combinatorial Species, Backtracking Algorithms, and Distributed Computation, 2008, http://www. sagemath.org/.

[23] N. Trinajstić, S. Nikolić, A. Miličević, I. Gutman, On Zagreb Indices , Kem. Ind. 59 (2010) 577-589 (in Croatian).

[24] B. Zhou, Zagreb Indices, MATCH Commun. Math. Comput. Chem. 52 (2004) 113-118.

[25] B. Zhou, Remarks on Zagreb Indices, MATCH Commun. Math. Comput. Chem. 57 (2007) 591-596.

[26] B. Zhou, I. Gutman, Further Properties of Zagreb Indices , MATCH Commun. Math. Comput. Chem. 54 (2005) 233-239. 